\title{
Toxic megacolon in ulcerative colitis: a continuing challenge
}

\author{
T. J. MusCrofT* \\ M.B., M.Sc., F.R.C.S. \\ P. AsQuith \\ M.D., F.R.C.P.
}

\author{
P. M. WARREN \\ M.B., M.R.C.P. \\ R. D. MONTGOMERY \\ M.D., F.R.C.P.
}

\author{
G. S. SoKHI* \\ M.B., F.R.C.S.
}

Departments of Medicine, and *Surgery, East Birmingham Hospital, Birmingham 9

\begin{abstract}
Summary
The incidence, predisposing factors, management and outcome of toxic megacolon (TM) have been reviewed in 65 cases of severe ulcerative colitis (UC) and compared in 2 successive 6-year periods before and after January, 1973. Nineteen episodes of TM occurred in 18 patients. Despite a conscious aim towards earlier surgery in recent years this was not achieved, and despite more intensive medical therapy the incidence of TM was unchanged. Emergency operative mortality in UC fell from $36 \%$ to $21 \%$ but the mortality of TM remained at $30 \%$. The chief cause of death was colonic perforation. Mortality was associated with increased age, longer pre-operative hospital stay and lower levels of serum albumin. These findings reemphasize the need for earlier surgery if TM is to be prevented, but such a policy must result in some unnecessary emergency colectomies.
\end{abstract}

\section{Introduction}

The prognosis of severe ulcerative colitis (UC) has improved in recent years, owing to increasing awareness of the value of prompt and intensive medical treatment in fulminating cases, with early recourse to surgery if improvement is not rapidly achieved or maintained (Lennard-Jones and Vivian, 1960; Goligher, Hoffman and de Dombal, 1970; Truelove and Jewell, 1974; Barany and Lindholmer, 1974). However, the predictive features, timing and long-term justification for emergency surgery are still debatable. The complication of toxic megacolon (TM) remains one of the gravest of medical emergencies; because of its rarity and varied presentation there are special problems in prevention, diagnosis and management. Causal factors have long been questioned, including the possible harmful effects of steroid or anticholinergic drugs, opiates, hypokalaemia, barium enemas or altered bowel flora. Extensive transmural spread of inflammation and muscle damage are constant features, sometimes with destruction of Auerbach's plexus (Sampson and Walker, 1961; Norland and Kirsner, 1969; Jalan et al., 1969).

Sixty patients are reviewed with severe UC presenting to a gastrointestinal unit, in order to compare clinical and laboratory features of those cases which were complicated by TM, and to evaluate progress in prevention and management.

\section{Patients and methods}

Nineteen episodes of TM occurred in 18 patients, in a series of 60 patients who suffered 65 attacks of severe UC during 1967-1979. Criteria for the diagnosis of TM were those of Jones and Chapman (1969) and the definition of severe UC was that of Truelove and Witts (1955). Thus all severe cases had numerically defined features of frequent bloody diarrhoea, tachycardia, pyrexia, anaemia and a high erythrocyte sedimentation rate (ESR). These represented $14 \%$ of all cases of UC, and TM occurred in $4.1 \%$. No cases in this series revealed any clinical or histological evidence of Crohn's disease. Details of age, length of history, pre-operative hospital time, preceding use of drugs, serum potassium, albumin, orosomucoid and ESR were analysed. Data were compared in patients with and without toxic megacolon, and also in 2 groups presenting in 6-year periods before and after January, 1973.

Standard intensive medical treatment was given, with intravenous rehydration and alimentation, infusions of blood and/or plasma, high doses of parenteral hydrocortisone and immediate oral sulphasalazine if the patient could tolerate it. Oral feeding was restarted within a few days, often with elemental diet supplements, and steroid enemas were administered as soon as they could be retained. Antibiotics were given only if there were specific 
TABLE 1. Mean indices in 65 cases of severe ulcerative colitis

\begin{tabular}{lccc}
\hline & $\begin{array}{c}\text { Severe UC } \\
\text { No operation } \\
\text { (35 cases) }\end{array}$ & $\begin{array}{c}\text { Severe UC } \\
\text { Emergency operation } \\
\text { (11 cases) }\end{array}$ & $\begin{array}{c}\text { TM } \\
\text { (19 cases) }\end{array}$ \\
\hline Age: years & 32 & 37 & 41 \\
Hb (g/dl) & $9 \cdot 8$ & $10 \cdot 2$ & $11 \cdot 3$ \\
Serum K (mmol/l) & $3 \cdot 8$ & $3 \cdot 4$ & $3 \cdot 5$ \\
Serum albumin (g/l)* & 30 & 33 & 27 \\
ESR (mm/hr) & 64 & 49 & 47 \\
Orosomucoid (mmol/l) $\dagger$ & 254 & 239 & 226 \\
\hline
\end{tabular}

* Data available in 58 cases.

† Data available in 37 cases.

indications. Abdominal girth was measured daily and the abdomen was X-rayed on admission and subsequently if the girth increased.

\section{Results}

\section{Predictive features}

In Table 1, data are summarized in 3 groups of cases of severe UC-those treated medically, those requiring emergency surgery (without TM), and those who developed TM. The overall figures show no significant differences between groups. However, detailed analysis shows that the serum albumin levels had prognostic value with regard to the need for emergency surgery, particularly in cases of TM

TABLE 2. Serum albumin levels related to outcome in 58 cases of severe ulcerative colitis

\begin{tabular}{lcc}
\hline & $\begin{array}{c}<30 \mathrm{~g} / 1 \\
(25 \text { cases }) \\
\%\end{array}$ & $\begin{array}{c}>30 \mathrm{~g} / 1 \\
(33 \text { cases }) \\
\%\end{array}$ \\
\hline Emergency operation & 36 & 18 \\
Toxic megacolon & 32 & 12 \\
Emergency operation mortality & 44 & 17 \\
Subsequent surgery & 12 & - \\
\hline
\end{tabular}

(Table 2). Of 25 patients whose serum albumin was less than $30 \mathrm{~g} / 1,48 \%$ ultimately required surgery, and of 11 with serum albumin less than $26 \mathrm{~g} / 1,8$ came to surgery ( 5 with TM). Conversely the respective albumin levels in the 3 patients who survived TM on medical treatment were 27,34 and $36 \mathrm{~g} / \mathrm{l}$.

The serum orosomucoid and ESR, although relevant to the severity of colitis, were of no predictive value within the series. Hypokalaemia was not a factor in the development of TM. In 10 cases the serum potassium was greater than $3.5 \mathrm{mmol} / \mathrm{l}$ at the time of onset of dilatation and in 3 cases (all in the later group) it was $4.0 \mathrm{mmol} / 1$. Patients with TM were on average 7 years older than the remainder. This difference was unrelated to the length of history. The mean age of fatal cases of TM was higher still
(56 years: range 42-74). No patients had received opiates or anticholinergic drugs and only 2 ha市 had a recent barium enema. Although corticosteroif therapy might affect the frequency of TM, it occurred in 2 cases not so treated. Stool isolates of Salmonellav Staphylococcus and Campylobacter were obtaine为 from several cases of severe UC, but none of these developed TM.

\section{Operative results}

Fourteen cases came to surgery after conservative measures failed and 4 died. Two of the survivors. had undergone 'less than colectomy' and both died after subsequent colectomy. The mean age in theo operative group was 43 years (39 years in survivers and 54 years in those who died). The mean preeo operative hospital stay of fatal cases was 20 dayss compared to 10 days in those surviving operation.

Seven patients had immediate ileostomy and pan? proctocolectomy $(\mathrm{I}+\mathrm{PPC})$ with 3 deaths. Ileostomy and subtotal colectomy $(\mathrm{I}+\mathrm{STC})$ was performed on $\overrightarrow{\mathrm{b}}$ 5 patients; one died and of the 4 survivors one required an urgent proctectomy for haemorrhage on the 11 th postoperative day and 2 others under went rectal excision later for continuing symptomso Two patients had 'less than colectomy'. One suf? fered a cardiac arrest at operation after 27 days of medical treatment. Following resuscitation, theplanned I + PPC was abandoned in favour of a loop ileostomy and venting colostomies in the transverse and sigmoid colon (Turnbull procedure). The patien? recovered, but died of sepsis following panproctoco $\rightarrow$ lectomy 4 months later. The second patient appeared. to have only distal dilatation and a transverse colostomy alone was performed. The acute episode settled, but continuing symptoms led to I+PPC
after which she died of prolonged sepsis.

Three patients who died postoperatively had alP suffered colonic perforation before surgery and alb succumbed to septic complications. The fourtho postoperative death was a 63-year-old woman who had a cardiac arrest at induction of anaesthesia 80 days after admission, and sustained irreversible cerebral damage. 
Comparison of early and recent series

In Tables 3 and 4 laboratory findings and clinical outcome are compared in the 2 consecutive series of patients with TM before and after January, 1973. In the recent series the mean serum potassium and albumin levels at the time of colonic dilatation were

TABlE 3. Mean indices in 19 cases of toxic megacolon compared in 6-year periods before and after January 1973

\begin{tabular}{lcc}
\hline & $\begin{array}{c}1967-73 \\
(9 \text { cases })\end{array}$ & $\begin{array}{c}1973-79 \\
(10 \text { cases })\end{array}$ \\
\hline Age (years) & 40 & 42 \\
Length of history (months) & 32 & 28 \\
Serum K (mmol/l) & $3 \cdot 2$ & 3.7 \\
Serum albumin (g/l) & 24 & 29 \\
Haemoglobin (g/l) & $11 \cdot 2$ & $11 \cdot 4$ \\
Pre-op. hospital time (days) & $11 \cdot 5$ & 14 \\
\hline
\end{tabular}

higher than in the early series. Nevertheless there was no apparent decline in the incidence of TM. In cases coming to surgery the mean duration of pre-operative in-patient treatment in the recent and early series showed no significant change. The operative mortality of emergency surgery in severe UC fell from $36 \%$ to $21 \%$, but the mortality rate of TM (with or without surgery) changed insignificantly from $33 \%$ to $30 \%$.

\section{Discussion \\ Diagnosis}

TM is a well known but rare complication of UC, with a reported incidence of between $1.6 \%$ and $3 \%$. The higher incidence of $4.1 \%$ in this series is due to the referral of some fulminating cases via the Regional Communicable Diseases Department of this hospital. Students seldom encounter the condition, and a family doctor may never see a case in his career, hence it is not surprising that diagnostic diffculties arise. The onset of colonic dilatation is sometimes insidious. Diarrhoea declines, the temperature may fall and the haemoglobin may be normal; for these reasons 3 cases in this series did not even fulfil the chosen diagnostic criteria of severe UC. Distension may be confined to the epigastrium. Although there is usually increase in pain and tenderness, the patient's judgment is often clouded, with mental changes varying from depression and withdrawal to confusion and euphoria.

\section{Prevention}

The improvement in biochemical parameters in the later series reflects more effective medical care in recent years, yet TM was not prevented and its mortality rate was undiminished. In spite of a conscious policy of earlier surgery in non-responding cases, this goal was not achieved. Only 2 cases in the first group and 4 in the recent group had surgery within 8 days of admission. This pre-operative delay is one definable factor linked to the continuing high incidence of TM in this series, and to the mortality of TM itself.

The reasons for delay are complex. In a large general hospital, patients with severe colitis may present in different departments with complicating conditions, leading to multiple referrals. An operation resulting in a permanent stoma is not lightly to be recommended, and the patient may refuse it. The emergency surgical mortality rate of $21 \%$ in this series compares with that of $27 \%$ at the Mayo Clinic, where no improvement was reported over 10 years (Von Heerden, McIlrath and Adson, 1978). Even when a policy decision has been made, there is reluctance to undertake such dangerous surgery while hope remains of improving the patient's pre-operative state. The decision can be eased by strict adherence to a predetermined timetable, but the exact timing of such a programme is still debatable (Goligher et al., 1970; Truelove and Jewell, 1974). A second problem is in assessing lack of progress in the first few days, when the clinical picture has been modified by high-dose steroid therapy, intravenous rehydration and transfusions. In addition to measurement of abdominal girth, daily abdominal X-rays are desirable, but in some hospitals this poses real logistic problems.

The prognostic value of serum albumin, prealbumin and C-reactive protein estimation was shown by Buckell et al. (1979) who emphasized the

TABLE 4. Mortality of emergency surgery in 25 cases of severe ulcerative colitis compared in 6-year periods before and after January 1973

\begin{tabular}{lcccc}
\hline & \multicolumn{2}{c}{$1967-1973$} & \multicolumn{2}{c}{$1973-1979$} \\
& Operations & Deaths & Operations & Deaths \\
\hline Toxic megacolon & 7 & 3 & 7 & 2 \\
Other severe ulcerative colitis & 4 & 1 & 7 & 1 \\
& 11 & 4 & 14 & 3 \\
\hline
\end{tabular}


hopeful outlook in cases with falling levels of Creactive protein and steady or rising albumin levels. It must be emphasized that TM is not an isolated phenomenon, but serves as a sign of impending disintegration of other parts of the colon (Brooke, 1968). Prognosis is unrelated to the diameter of dilatation (Norland and Kirsner, 1969) and perforation almost invariably occurs distally to the dilated segment.

It is claimed in support of a policy of early surgery that the long-term prognosis of severe UC is poor with regard to renewed risks and the quality of life. In the present series of 60 patients, 35 survived their first severe attack without surgery. Nine subsequently had surgery (4 as emergencies) for severe relapses or intractable symptoms. Twenty-six patients $(74 \%)$ continue under medical surveillance after 2-13 years and many of those are symptom-free. These figures do not clearly point to any long-term as opposed to short-term benefit from earlier emergency surgery.
Management

Although early surgery can reduce the inciden of TM, authors differ in their management of TM once it has developed. Mortality rates ranging from $6 \%$ to $46 \%$ have been recorded (Brooke, 196\% Norland and Kirsner, 1969; Jalan et al., 196\% Goligher et al., 1970; Binder, Patterson and Gloyze 1974). The usual advice is for surgery within $72 \mathrm{~h}$ and there is extreme urgency if plain X-rays revegas-filled undermined ulcers and mucosal islangs (Brooke, 1968) (Fig. 1). Norland and Kirsner (1969) found that operation was invariable if $T M$ pe $\vec{B}$ sisted for 12 days. In the present series 14 out of $1 \frac{8}{q}$ patients came to emergency surgery; this compares with 17 out of 18 patients described by Binder et at (1974) and all 17 cases reviewed by Scott et as (1974).

The long-term outlook for patients surviving TM with medical measures is worse than for othes patients with severe UC. Sirinek et al. (197)

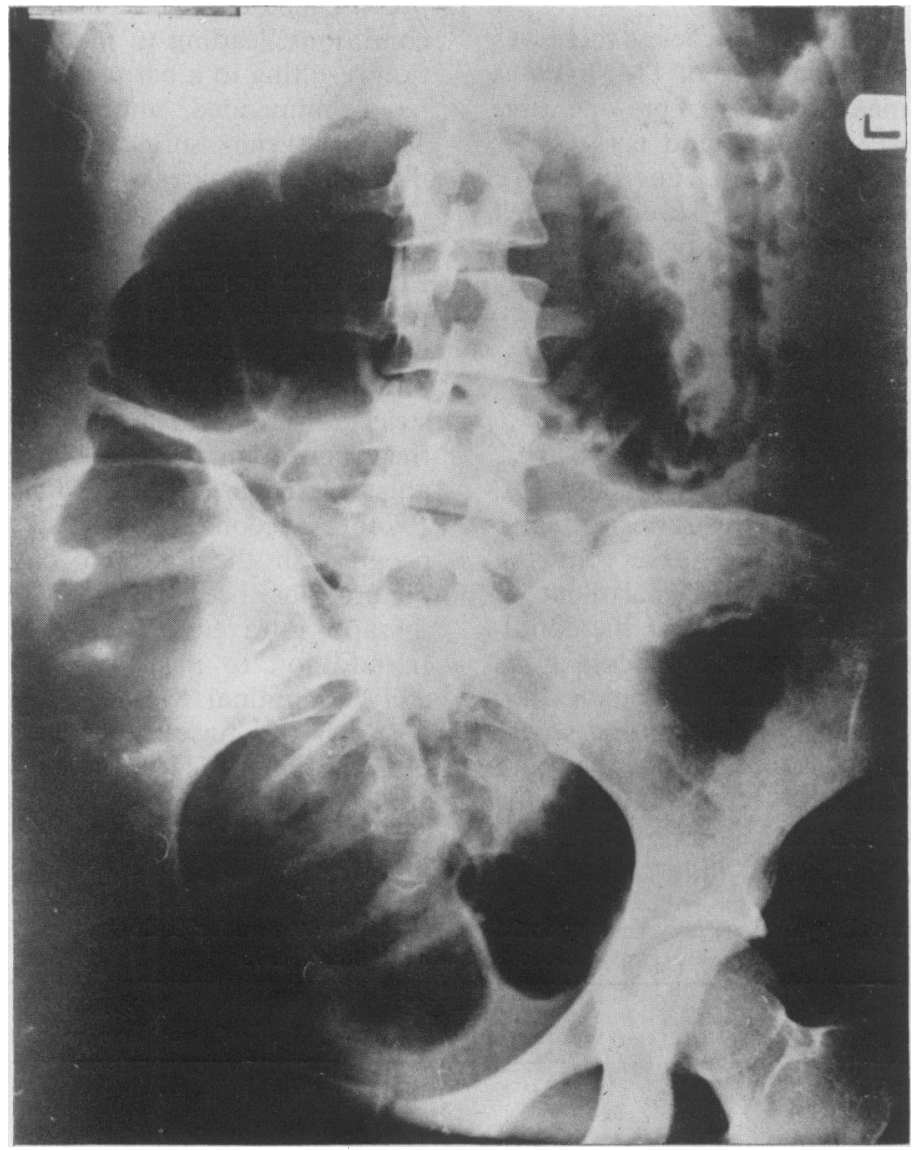

Fig. 1. Toxic megacolon showing undermined transmural ulceration and mucosal islands. Immediate panproctocolectomy led to an uncomplicated recovery. 
reviewed 42 patients with TM. Of 11 treated medically, 2 died, 5 had subsequent colectomies, 3 had multiple exacerbations needing hospital treatment, and only one remained asymptomatic. It is clear that medical treatment should be abandoned at an early stage in the presence of TM. Perhaps the only exception would be the case without all the accepted parameters of severity, with a serum albumin level greater than $30 \mathrm{~g} / 1$ and falling levels of C-reactive protein.

With regard to the type of operation, good results have been reported using the Turnbull procedure of loop ileostomy and venting colostomy (Shipp, 1974; Fazio, Turnbull and Goldsmith, 1976), but this necessitates a secondary colectomy, whereas with immediate colectomy increasingly low mortality rates have been achieved (Binder et al., 1974; Strauss et al., 1976; Sirinek et al., 1977). The case for immediate rectal excision is controversial. In severely ill patients a proctectomy may be an intolerable additional stress; however, the rectum is almost always severely involved and may require later removal, as was the case in 3 of 4 survivors in the present series who originally underwent I+STC. There has been recent interest in ileorectal anastomosis following emergency colectomy, even in the presence of rectal disease (Watts and Hughes, 1976; Kubachandi et al., 1976).

\section{Conclusions}

The significance of TM is that it is one sign of disintegrating colon, and from its nature it is unlikely that its mortality can be substantially reduced. Attention must therefore be directed to its prevention. The serum albumin has been shown to be of some prognostic value, in so far as cases with the lowest levels have a $40 \%$ chance of developing TM and a $70 \%$ chance of requiring surgery. Apart from the day-to-day behaviour of the acute-phase serum proteins, no other predictive tests are known, and the problem remains primarily clinical.

The principle of earlier emergency surgery has been widely accepted in theory, but the practical difficulties are formidable and the authors believe that their experience is far from unique. A medicosurgical audit in an individual hospital, such as was undertaken in the present study, should lead to closer co-ordination and improved results. There is no doubt, however, that a stricter plan of early operation must lead to an increased number of unnecessary emergency colectomies. This is the price which would have to be paid for the total prevention of TM.

\section{Acknowledgment}

We are indebted to our surgical colleagues, Mr A. B. M. Currie, Mr M. D. Middleton and Mr R. W. Tudor for per- mission to publish details of cases which came under their care.

\section{References}

BARANY, F. \& LINDHOLMER, B. (1974) Importance of early intensive treatment in severe attacks of ulcerative colitis. Rendiconti di Gastroenterologia, 6, 174.

Binder, S.C., PatTerson, J.F. \& Gloyzer, D.J. (1974) Toxic megacolon in ulcerative colitis. Gastroenterology, 66, 909.

BROOKE, B.N. (1968) Indications for emergency colectomy in ulcerative colitis. Diseases of the Colon and Rectum, 11, 85.

Buckell, N.A., Lennard-Jones, J.E., Hernandez, M.A., KhaN, J., Riches, P.G. \& WADSWORTH, J. (1979) Measurement of serum proteins during attacks of ulcerative colitis as a guide to patient management. Gut, 20, 22.

FAzio, V., Turnbull, R.B. \& Goldsmith, M. (1976) Ileostomy-colostomy for the megacolon phase of toxic ulcerative colitis. In: Gastrointestinal Emergencies (Ed by Clearfield, H.R. \& Dinoso, V.P.). Grune and Stratton, New York.

Goligher, J.C., Hoffman, D.C. \& DE Dombal, F.T. (1970) Surgical treatment of severe attacks of ulcerative colitis, with special reference to the advantages of early operation. British Medical Journal, 4, 703.

JAlan, K.N., Sircus, W., Card, W.I., Falconer, C.W.A. \& BRUCE, J. (1969) An experience of ulcerative colitis 1. Toxic dilatation in 55 cases. Gastroenterology, $51,68$.

Jones, J.H. \& Chapman, M. (1969) Definition of megacolon in colitis. Gut, 10, 562 .

Kubachandi, I.T., Trimpi, H.D., Sheets, J.A., Stasik, J.J. \& KLECKNER, F.S. (1976) Ileorectal anastomosis for ulcerative and Crohn's colitis. American Journal of Surgery, $135,751$.

LenNARD-Jones, J.E. \& Vivian, A.B. (1960) Fulminating ulcerative colitis: recent experience in management. British Medical Journal, 2, 96.

Norland, C.C. \& KirSNER, J.B. (1969) Toxic dilatation of the colon (toxic megacolon): etiology, treatment and prognosis in 42 patients. Medicine. Baltimore, 48, 229.

SAMPSON, P.A. \& WALKeR, F.C. (1961) Dilatation of the colon in ulcerative colitis. British Medical Journal, 2, 1119.

ScotT, H.W., SAWYers, J.L., Gobbel, W.G., Graves, H.A. $\&$ SHull, D.J. (1974) Surgical management of toxic dilatation of the colon in ulcerative colitis. Annals of Surgery, 179, 647.

SHIPP, J.D. (1974) Surgery for toxic megacolon: experience with the Turnbull-Weaklet technique. Diseases of the Colon and Rectum, 17, 342.

SirineK, K.R., TetiRick, C.E., Thomford, N.R. \& PACE, W.G. (1977) Total proctocolectomy and ileostomy: procedure of choice for acute toxic megacolon. Archives of Surgery, 112, 518.

Strauss, R.J., Flint, G.W., Platt, N., Levin, L. \& Wise, L. (1976) The surgical management of toxic dilatation of the colon. Annals of Surgery, 184, 682.

TRUelove, S.C. \& WITTS, L.J. (1955) Cortisone in ulcerative colitis: final report on a therapeutic trial. British Medical Journal, 2, 1041.

Truelove, S.C. \& Jewell, D.P. (1974) Intensive intravenous regimen for severe attacks of ulcerative colitis. Lancet, $i$, 1067.

Von Heerden, J.A., McIlrath, D.C. \& Adson, M.A. (1978) The surgical aspects of chronic mucosal inflammatory bowel disease (chronic ulcerative colitis). Annals of Surgery, 187, 536.

WATTS, J. McK. \& Hughes, E.S.R. (1976) Ulcerative colitis and Crohn's disease: result after colectomy and ileorectal anastomosis. British Journal of Surgery, 64, 77. 